\title{
Surface figure measurements of radio telescopes with a shearing interferometer
}

\author{
E. Serabyn, T. G. Phillips, and C. R. Masson
}

\begin{abstract}
A new technique for determining the surface figure of large submillimeter wavelength telescopes is presented, which is based on measuring the telescope's focal plane diffraction pattern with a shearing interferometer. In addition to the instrumental theory, results obtained using such an interferometer on the 10.4-m diam telescope of the Caltech Submillimeter Observatory are discussed. Using wavelengths near $1 \mathrm{~mm}$, a measurement accuracy of $9 \mu \mathrm{m}$, or $\lambda / 115$, has been achieved, and the rms surface accuracy has been determined to be just under $30 \mu \mathrm{m}$. The distortions of the primary reflector with changing elevation angle have also been measured and agree well with theoretical predictions of the dish deformation. Key words: Shearing interferometry, radio telescopes, radio holography.
\end{abstract}

\section{Introduction}

As radio telescopes are pushed to ever higher frequency operation, the constraints on their surface accuracies become correspondingly more severe. For operation at $900 \mathrm{GHz}$, the highest radio frequency at which mountaintop astronomical observations are feasible, $\lambda / 10$ wavefront accuracy requires a surface root mean square (rms) error of $17 \mu \mathrm{m}$. Since this surface error includes contributions not only from static errors but also from elevation angle and temperature dependent distortions of the reflector, the achievement of such accuracies is a goal which places severe demands not only on the telescope design but also on the techniques used to measure and set the reflector surface. Consequently, no currently operational radio-astronomical telescope has a surface accuracy of better than $\sim 30-\mu \mathrm{m}$ rms.

The new Caltech Submillimeter Observatory ${ }^{1}$ (CSO) provides a case in point. This telescope, located near the summit of Mauna Kea on the island of Hawaii, is intended for operation in the $200-900-\mathrm{GHz}$ frequency range. The telescope consists of a segmented 10.4-m diam parabolic primary reflector and a 1.02$\mathrm{m}$ diam hyperbolic secondary reflector arranged in a

C. R. Masson is with Harvard-Smithsonian Center for Astrophysics, 60 Garden Street, Cambridge, Massachusetts 02138; the other authors are with California Institute of Technology, Division of Physics, Mathematics, and Astronomy, Pasadena, California 91125.

Received 6 March 1990.

0003-6935/91/101227-15\$05.00/0.

(C) 1991 Optical Society of America.
Cassegrain configuration, with a final focal ratio or $F /$ No. of 6.5. Since the surfaces of the eighty-four individual panels making up the primary reflector are accurate to 5-10- $\mu \mathrm{m} \mathrm{rms,} \mathrm{the} \mathrm{surface} \mathrm{errors} \mathrm{are} \mathrm{expected}$ to be dominated by panel setting and gravitational deformation errors. Mechanical measurements indicate that the primary has an rms surface error of $\sim 27$ $\mu \mathrm{m}$ at present, but the ultimate goal is to reduce this error to $\sim 15 \mu \mathrm{m}$.

To date, the surface figures of high frequency radio telescopes have been measured by a number of related techniques, commonly referred to as radio holography. ${ }^{2-4}$ Where two similar telescopes are available, such as two elements of an interferometric array, one of the telescopes is used as a reference, by pointing it directly at a distant point source, while the telescope to be measured is tipped off-source to map out its far field pattern. ${ }^{3}$ The electric field response of the second telescope to the distant off-axis source is determined by correlating its signal with that of the reference telescope at points on a rectangular $N \times N$ grid surrounding the source. Since the far field pattern corresponds to the Fraunhofer diffraction limit, ${ }^{5}$ this grid of measurements is then numerically Fourier transformed to yield the electric field distribution in the aperture plane of the telescope under inspection. The phase of the aperture plane field is then related geometrically to the deviations of the surface from its ideal parabolic shape.

If only one telescope is available, alternative techniques include installing a second, much smaller reference telescope nearby, ${ }^{2,6}$ or mounting an upward looking reference feed horn behind the secondary mirror, 7,8 which views the source directly along the telescope 
axis. Because the collecting area of the reference system is much smaller than that of the telescope itself, its response will be quite weak, necessitating a strong source, such as a satellite, ${ }^{6}$ a nearby transmitter, ${ }^{7}$ or an astronomical maser. ${ }^{8}$

Although all these variations work quite well, they all suffer from the practical drawback that two receivers, and in some cases two entire telescopes, must be available. On the other hand, the technique of phaseless holography, ${ }^{4,9}$ which reconstructs the aperture plane phase from a comparison of far field power maps obtained with the telescope in and out of focus, requires only one receiver. However, because of the high signal-to-noise ratio required by this technique, ${ }^{10}$ a strong, and therefore nearby transmitter is called for. Since such a transmitter would necessarily be located on a nearby mountaintop, dish measurements are possible only at a single, near-horizon elevation angle.

Because it would clearly be advantageous to use only one receiver and to measure the dish surface as a function of elevation angle, an alternate method of performing holographic surface measurements was sought. Ideally, the measurement technique adopted should work at a frequency for which the telescope already has a receiver (in the case of the CSO, near or shortward of $\lambda=1 \mathrm{~mm}$ ). This constraint eliminates any techniques which rely on satellite transmitters, since their frequencies of operation are much too low $(<40 \mathrm{GHz})^{6,11}$ Furthermore, since mountaintop transmitters are also ruled out by the desire to measure the dish surface over the full range of elevation angles, sufficient sensitivity is required to allow the use of an astronomical source. A final desirable characteristic is variable frequency of operation so that, as the surface is improved, the measurement wavelength can also be shortened.

In the following, we describe a new holographic measurement technique which meets all these criteria and is particularly suited for the high frequencies of the CSO. The succeeding sections outline the theory of operation of such a device, describe an instrument designed along these lines, and present measurements obtained using the instrument on the CSO.

\section{The Concept}

\section{A. Spatial Measurements}

The new measurement technique presented here arises from an analogy with the two-telescope interferometric method, wherein the two distinct telescope reflectors are replaced by two images of a single primary mirror. The optical arrangement used to produce these images is shown in Fig. 1. The collimated near millimeter wavelength radiation from a distant point source is first focused by the telescope to the Cassegrain focal plane, and thereafter reexpands to a tertiary off-axis paraboloid $(P 1)$. $P 1$ recollimates the radiation and images the primary mirror slightly more than one focal length beyond itself. A dielectric beam splitter (BS), inserted into the beam just before the image of the primary, splits the radiation polarized normal to

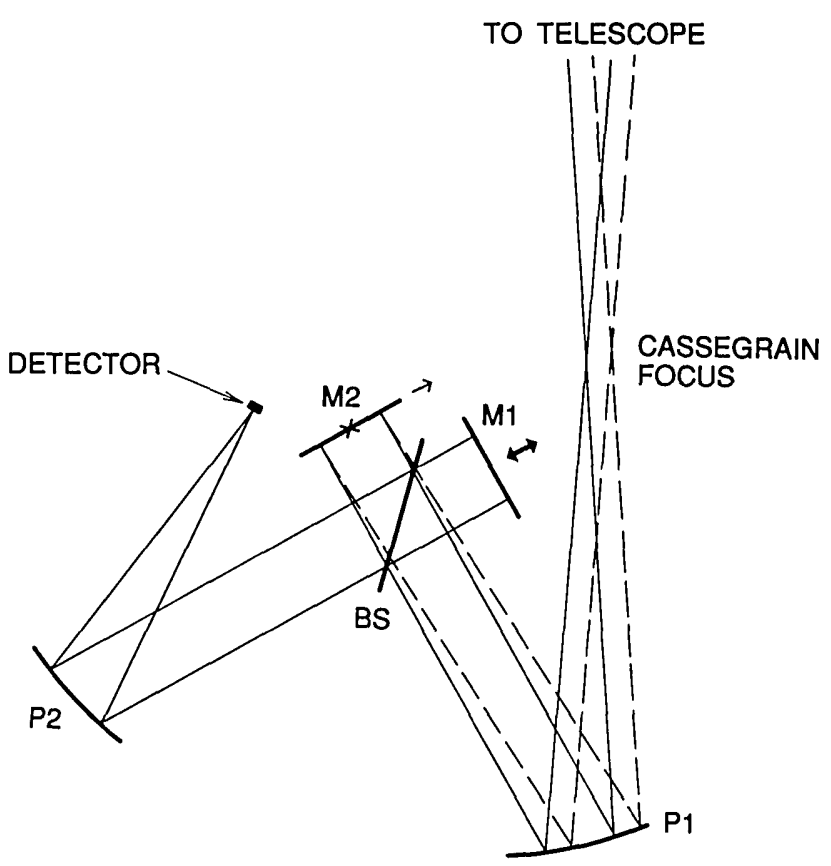

Fig. 1. Schematic layout of the interferometer optics: $P 1$ and $P 2$, off-axis paraboloids; $B S$, Mylar beam splitter; and $M 1$ and $M 2$, plane mirrors. The double arrow shows the direction of travel of $M 1$, and the small arrow and cross, the rotation axes of $M 2$. The light solid lines indicate the on-axis beam from $M 1$, and the dashed lines the off-axis beam from $M 2$.

the plane of incidence into two approximately equal parts $^{11}$ (the second polarization will be ignored hereafter), resulting in the creation of two equivalent images of the primary. Plane mirrors $M 1$ and $M 2$, positioned at these image surfaces, reflect the radiation back to the beam splitter, where the radiation recombines before being focused onto a detector by off-axis paraboloid $P 2$.

Although this optical setup constitutes a TwymanGreen interferometer, ${ }^{12}$ which is a standard instrument for optical surface testing, our point of departure is rather different from that of the normal optical usage for two reasons. First, at near millimeter wavelengths, there are no arrays capable of measuring pupil plane fringe patterns directly, so that a single detector must be used to perform point-by-point measurements. Second, since the normal radio approach is to use a wavelength much greater than the maximum wavefront error, there is no $2 \pi$ phase ambiguity, while in the optical regime, the counting of fringes allows the wavelength to be shorter than the path length errors. The standard Twyman-Green interferometer must consequently be modified for our purposes.

Consider therefore a rotation of mirror $M 2$ out of the plane normal to the collimated beam impinging on it. In a geometric optics viewpoint, such a rotation steers the beam from the detector off-axis in the Cassegrain focal plane, and so on the sky also (Fig. 1). Since $M 2$ is coincident with an image of the primary, a rotation of $M 2$ is thus equivalent, in the paraxial approximation, to steering the telescope off source. The image mir- 
rors $M 1$ and $M 2$ are then analogous to the two telescopes in the standard interferometric holography technique, since from the point of view of the detector, mirror $M 1$ directs a reference beam toward the source, while mirror $M 2$ is used to steer a second beam off-axis.

Although this simple geometric description allows for easy visualization, it is obviously incomplete, since diffraction is the ultimate cause of the far field pattern $^{13}$ which we wish to measure. We, therefore, turn to a discussion of the interferometer which includes the effects of diffraction but limit ourselves to the paraxial case, assuming thereby that all $F /$ Nos. in the system are large, and all off-axis angles small. Although the $F /$ Nos. in the interferometer constructed are 6.5 at the input and 4 at the output, a discussion of the effects of moderate $F /$ Nos. is deferred to a later paper, as is the related question of how far off-axis it is safe to measure.

In the paraxial approximation, the incoming radiation from an on-axis far field point source $\left(2 D^{2} / \lambda=216\right.$ $\mathrm{km}$ at $\lambda=1 \mathrm{~mm}$ ) produces a diffraction pattern $E_{c}\left(x_{c}, y_{c}\right)$ in the Cassegrain focal plane which is the 2-D Fourier transform of the aperture plane field. ${ }^{14}$ Here $E_{c}$ is the complex electric field amplitude, and $x_{c}$ and $y_{c}$ are two orthogonal focal plane coordinates. Furthermore, in the small angle limit, the far field pattern $E_{f f}\left(\phi_{x}, \phi_{y}\right)$ is also the Fourier transform of the aperture plane field. ${ }^{5}$ Here $\phi_{x}$ and $\phi_{y}$ are small angles in the sky measuring displacements from the telescope axis in two orthogonal directions. The far field and focal plane fields are thus proportional, i.e.,

$$
E_{c}\left(x_{c}, y_{c}\right)=C E_{f f}\left(\phi_{x}, \phi_{y}\right) \text {. }
$$

Here $C$ is a constant, and the sky and focal plane coordinates are related via $x_{c}=f_{e q} \phi_{x}$ and $y_{c}=f_{e q} \phi_{y}$, where $f_{e q}$ is the focal length of the equivalent paraboloid. ${ }^{15}$ A measurement of the telescope's Cassegrain focal plane pattern is thus equivalent to measurement of its far field. With this understanding, the proposed interferometer can be viewed as a device to examine the telescope's focal plane pattern. Note that this is the opposite of normal radio procedure, wherein the telescope is tipped off-source and the receiver kept onaxis; here the source is kept on-axis, and the focal plane field sensed off-axis.

Assuming that the optical elements in the interferometer have apertures large enough to neglect further diffraction, and that the instrument itself introduces no aberrations (a good assumption since $P 1$ and $P 2$ are oriented so as to partially cancel each other's coma ${ }^{16}$ ), the interferometer will then transfer two scaled images of the Cassegrain diffraction pattern onto the instrumental output focal plane. With mirrors $M 1$ and $M 2$ both oriented normal to the radiation incident on them, each arm of the interferometer relays to the output plane the same field distribution:

$$
E_{o}\left(x_{o}, y_{o}\right)=\frac{1}{2} \frac{F_{c}}{F_{o}} E_{c}\left(x_{c}, y_{c}\right)
$$

where the linear coordinates $x_{o}, y_{o}$ in the output focal plane are related to those in the Cassegrain focal plane by $x_{o}=\left(F_{o} / F_{c}\right) x_{c}$ and $y_{o}=\left(F_{o} / F_{c}\right) y_{c}$, with $F_{c}$ and $F_{o}$ the Cassegrain and output $F /$ Nos., respectively. The factor $1 / 2$ arises from the assumption of a $50-50$ beam splitter.

In the paraxial approximation, a rotation of mirror M2 simply translates the diffraction pattern produced by arm 2 of the interferometer laterally in the output focal plane to yield the superposition of the two electric fields:

$$
E_{o}\left(x_{o}, y_{o}\right)=\frac{1}{2} \frac{F_{c}}{F_{o}}\left[E_{c}\left(x_{c}, y_{c}\right)+E_{c}\left(x_{c}+2 f_{1} \theta_{x}, y_{c}+2 f_{1} \theta_{y}\right)\right],
$$

where $\theta_{x}$ and $\theta_{y}$ are the small rotation angles which mirror $M 2$ makes with the $x$ and $y$ axes, and $f_{1}$ is the focal length of $P 1$. A pointlike detector situated at the center of the diffraction pattern produced by the reference arm of the interferometer then sees, for monochromatic radiation and fixed $\theta_{x}, \theta_{y}$,

$$
E_{o}(0,0)=\frac{1}{2} \frac{F_{c}}{F_{o}}\left[E_{c}(0,0)+E_{c}\left(2 f_{1} \theta_{x}, 2 f_{1} \theta_{y}\right)\right] .
$$

Since $E_{c}$ is proportional to $E_{f f}$ by Eq. (1), and the $\phi$ and $\theta$ terms are related in the paraxial approximation via $f_{e q} \phi_{x}=2 f_{1} \theta_{x}$ and $f_{e q} \phi_{y}=2 f_{1} \theta_{y}$, we have

$$
E_{o}(0,0)=C^{\prime}\left[E_{f f}(0,0)+E_{f f}\left(\phi_{x}, \phi_{y}\right)\right] \text {, }
$$

with $C^{\prime}$ a new constant. Since the detector sees $E_{f f}\left(\phi_{x}, \phi_{y}\right)$ in addition to $E_{f f}(0,0)$, the rotation of mirror M2 is indeed equivalent to steering the telescope offsource.

\section{B. Spectral Measurements}

Because the far field pattern of the telescope is a function of frequency, it is necessary to restrict the spectral bandwidth reaching the detector. Current practice in the usual forms of radio holography is to use heterodyne receivers, which necessarily restrict the bandwidth sufficiently. However, how narrow a band is actually required? Considering a simply Airy pattern for the moment, the far field pattern will only be well defined over the entire region examined, if for all frequencies in the instrumental bandpass, the position of the outermost measured ring (or sidelobe) of the far field pattern does not vary by more than about half of the width of the ring. If measurements are made out to the $n$th sidelobe, the required condition is approximately

$$
\frac{n \Delta \lambda}{D} \leqslant \frac{\lambda}{2 D}
$$

where $D$ is the telescope diameter, $\lambda$ is the wavelength of the radiation, and $\Delta \lambda$ is its bandwidth. The necessary resolving power, $R(\equiv \lambda / \Delta \lambda)$, thus need only satisfy $R \gtrsim 2 n$, implying that a modest resolving power of the order of 20 is sufficient. (A more exact requirement is derived in Sec. III.C.)

Consequently, a resolving power as high as that provided by heterodyne receivers is not necessary. There is also no need for the phase measurement capability inherent in the heterodyne technique, because only relative phases are needed, and these are provided by 
the interferometer. It is therefore feasible to use a broadband detector, such as a bolometer with a suitably restricted bandpass, bringing with it a major advantage in terms of extra sensitivity. The signal-tonoise ratio (SNR) attainable by direct and heterodyne measurements of a broadband source in the far field can be compared via ${ }^{17}$

$$
\frac{\mathrm{SNR}_{d}}{\mathrm{SNR}_{h}}=\frac{2 k T_{n}}{\mathrm{NEP}} \frac{\Delta \nu_{d}}{\sqrt{\Delta \nu_{h}}},
$$

where $k$ is Boltzmann's constant, $T_{n}$ is the heterodyne receiver's system noise temperature, ${ }^{13}$ and NEP is the noise equivalent power of the direct detector. ${ }^{18}$ The subscripts $d$ and $h$ refer to corresponding direct and heterodyne parameters, such as the frequency bandwidths $\Delta \nu_{d}$ and $\Delta \nu_{h}$. Inserting typical values for these parameters at $\lambda=1 \mathrm{~mm}, T_{n} \sim 1000 \mathrm{~K}, \mathrm{NEP} \sim 10^{-14} \mathrm{~W} /$ $\mathrm{Hz}^{1 / 2}$ (both referred to above the atmosphere), $\Delta \nu_{d} \sim$ $100 \mathrm{GHz}$, and $\Delta \nu_{h} \sim 100 \mathrm{MHz}$, we see that $\mathrm{SNR}_{d} / \mathrm{SNR}_{h}$ $\approx 28$ for a broadband source in the far field. (Use of a narrowband near field source would increase the heterodyne SNR, but in this case, the flexibility to measure at different zenith angles is lost.)

Rather than using a narrowband filter to achieve the desired resolution, it is possible to use the interferometer itself, since it can also function as a Fourier transform spectrometer (FTS), by translating $M 1$ along the normal to its surface. With $M 2$ oriented to view a fixed off-axis angle, the superposition of the on- and off-axis electric fields then generates a cross-power interferogram which can be Fourier transformed to yield the response of the telescope at that off-axis angle as a function of frequency. The use of the interferometer as a spectrometer then lowers the requirement on the resolving power of the detector itself even further, and a broadband detector with a resolving power of a few suffice. The actual spectral resolution of the far field measurements is determined, ${ }^{19}$ as usual, by maximum path difference $L$, attained by the scanning mirror. For a two-sided interferogram with no apodization, $R=2 L / \lambda$, implying a required mirror travel of only a centimeter or so.

To measure a surface deviation of height $\epsilon$ on the dish, using radiation of wavelength $\lambda$, the SNR required with both beams centered on-source is approximately ${ }^{3} N \lambda / 4 \pi \epsilon$, where $N$ is the number of linear resolution elements across the dish. At a wavelength of 1 $\mathrm{mm}$, to achieve a horizontal resolution of $1 \mathrm{~m}$ and a vertical resolution of $10 \mu \mathrm{m}$, a SNR of $\sim 80$ is required. Modifying the formula for the SNR achievable by a scanning FTS ${ }^{20}$ for the case of source power given in units of antenna temperature ${ }^{13} T_{A}$ yields, for one polarization, scan time $\delta t$ and resolution $\Delta \nu$ :

$$
\mathrm{SNR}=\frac{k T_{A} \Delta \nu}{\mathrm{NEP}} \sqrt{\frac{\delta t}{4}} .
$$

Since $\delta t=L / v$, where $v$ is the scan velocity and $L=R \lambda$ / 2 , we get

$$
\mathrm{SNR}=\frac{k T_{A}}{\mathrm{NEP}} \sqrt{\frac{c \Delta \nu}{8 v}},
$$

where $c$ is the speed of light. Assuming $v=1 \mathrm{~cm} / \mathrm{s}$ and $\Delta v=15 \mathrm{GHz}$, we see that SNR $\approx 10 T_{A}$, with $\delta t=1.5$. To achieve the required SNR with a single scan of the FTS, the source strength must then satisfy $T_{A}>8 \mathrm{~K}$ at $\lambda=1 \mathrm{~mm}$. Such an SNR can readily be obtained on several of the planets with a bolometer, but other astronomical sources are too weak.

\section{Relationship to Existing Techniques}

Neglecting for the moment the spectroscopic properties of the instrument, the interferometer described is similar in several regards to shearing and wavefront tilting interferometers used in the optical and near IR regions. ${ }^{12,21}$ These are usually designed to examine the aberrated wavefronts in the pupil plane, since complete interference patterns can easily be seen visually or with infrared cameras. The novelty of the proposed interferometer thus lies in its examination of the focal plane diffraction pattern instead of the pupil plane and in its polychromatic capability. Both capabilities of these interferometers have previously been addressed in a general discussion of the theory of plane mirror interferometers. ${ }^{22}$

Direct measurement of a telescope's focal plane pattern has previously been proposed ${ }^{23}$ through measurements with separate microwave horns located on- and off-axis in the focal plane. However, this earlier approach suffers from the practical difficulty that, for the region nearest the optical axis, both horns need to occupy the same physical volume. This problem is obviated in our interferometer since there is only one detector.

Although the wavefronts from the source are actually tilted as a result of rotating mirror $M 2$, it is possible to consider the primary as the object under inspection, and so come to a slightly different viewpoint. Since the primary is imaged onto $M 2$, the wavefronts from a point located on the primary surface are sheared laterally by a rotation of $M 2$. We have therefore chosen the term shearing holography as a descriptive title for the new measurement technique presented here.

\section{Theory}

\section{A. Combined Spatial and Spectral Measurements}

Turning now to a more detailed theory of the interferometer, we allow for a path difference $\delta$ between the two arms of the interferometer. For monochromatic radiation, a pointlike detector situated at the center of the diffraction pattern from the reference arm of the interferometer then sees a field (neglecting constants of proportionality):

$$
E_{o}\left(x_{o}=0, y_{o}=0, \delta, k\right)=t(k)\left[E_{f f}(0,0, k) \exp (i k \delta)+E_{f f}\left(\phi_{x}, \phi_{y}, k\right)\right],
$$

where $k$ is the wavenumber of the radiation, the first term in the square brackets is the on-axis response delayed by $\delta$, the second term is the off-axis response, and $t(k)$ is the amplitude transmission function through the entire system of sky, telescope, interfer- 
ometer, and filters. Although $t(k)$ depends somewhat on the small angles $\phi_{x}$ and $\phi_{y}$, this is neglected here.

The power from a polychromatic source which hits the detector for a given $\delta, \phi_{x}$, and $\phi_{y}$ is then

$$
I\left(\phi_{x}, \phi_{y}, \delta\right)=\int_{0}^{\infty} T(k)\left|E_{f f}(0,0, k) \exp (i k \delta)+E_{f f}\left(\phi_{x}, \phi_{y}, k\right)\right|^{2} d k,
$$

with $T(k)=|t(k)|^{2}$. Expanding this expression,

$$
\begin{aligned}
I\left(\phi_{x}, \phi_{y}, \delta\right)=\int_{0}^{\infty} T(k)\left\{\left|E_{f f}(0,0, k)\right|^{2}+\left|E_{f f}\left(\phi_{x}, \phi_{y}, k\right)\right|^{2}\right. \\
\left.+2 \mathcal{R}\left[E_{f f}^{*}(0,0, k) E_{f f}\left(\phi_{x}, \phi_{y}, k\right)^{-i k \delta}\right]\right\} d k,
\end{aligned}
$$

where ${ }^{*}$ and $\mathscr{R}$ indicate the complex conjugate and real part, respectively. The first two terms on the righthand side of this equation are independent of $\delta$ and can be recast in terms of $P(0,0)=\int_{0}^{\infty} T(k)\left|E_{f f}(0,0, k)\right|^{2} d k$ and $P\left(\phi_{x}, \phi_{y}\right)=\int_{0}^{\infty} T(k)\left|E_{f f}\left(\phi_{x}, \phi_{y}, k\right)\right|^{2} d k$, which are the frequency integrated powers which would be measured with both mirrors aligned to look down the axis of the telescope, and both oriented to view the off-axis angles $\phi_{x}, \phi_{y}$, respectively. Extending the spectra as usual ${ }^{5}$ to negative $k$, we thus get

$$
\begin{aligned}
I\left(\phi_{x}, \phi_{y}, \delta\right)= & P(0,0)+P\left(\phi_{x}, \phi_{y}\right) \\
& +\int_{-\infty}^{\infty} T(k) E_{f f}^{*}(0,0, k) E_{f f}\left(\phi_{x}, \phi_{y}, k\right) \exp (-i k \delta) d k .
\end{aligned}
$$

The third term in this equation is the only one which is a function of $\delta$ and is also the only one which is linearly dependent on the off-axis field. This oscillatory function of $\delta$, the cross-power interferogram, is similar to a normal interferogram, except that the interfering fields arise in different parts of the input field. If we consider the input field to be the diffraction pattern produced by a point source, the two superimposed fields are fully coherent. Since the actual sources used were planets, which have finite extent, the effects of partial coherence are addressed in Appendix B.

Using a rapid scan interferometer with ac coupling, the first two terms in $I$ disappear, and the measured quantity $I_{m}\left(\phi_{x}, \phi_{y}, \delta\right)$ will consist solely of the crosspower term:

$$
I_{m}\left(\phi_{x}, \phi_{y}, \delta\right)=\int_{-\infty}^{\infty} T(k) E_{f f}^{*}(0,0, k) E_{f f}\left(\phi_{x}, \phi_{y}, k\right) \exp (-i k \delta) d k .
$$

This interferogram can be spectrally Fourier transformed to obtain the kernel of the integral and then normalized by $T(k) E_{f f}^{*}(0,0, k)$ to obtain the desired offaxis response $E_{f f}\left(\phi_{x}, \phi_{y}, k\right)$. However, normalizing instead by $S(k) \equiv T(k) E_{f f}^{*}(0,0, k) E_{f f}(0,0, k)$, which is directly measurable, is more useful. $S(k)$, which is the product of the source power spectrum and the system transmission, can be obtained from Eq. (14) by inverting the interferogram obtained with $M 2$ aligned onaxis or

$$
S(k)=\int_{-\infty}^{\infty} I_{m}(0,0, \delta) \exp (i k \delta) d \delta
$$

Using this result, we get the telescope's relative offaxis far field response as a function of $k$ :

$$
r\left(\phi_{x}, \phi_{y}, k\right) \equiv \frac{E_{f f}\left(\phi_{x}, \phi_{y}, k\right)}{E_{f f}(0,0, k)}=\frac{1}{S(k)} \int_{-\infty}^{\infty} I_{m}\left(\phi_{x}, \phi_{y}, \delta\right) \exp (i k \delta) d \delta .
$$

In the ideal case, $S(k)$ is the product of two real quantities, the source power spectrum and the system transmission, and so division by $S(k)$ should not affect the far field phases. However, errors which arise both from the uncertainty in determining the position of zero path difference within the interferometer ${ }^{19}$ and in pointing errors of the telescope, render division by $S(k)$ necessary in practice. For example, off-center sampling of the interferogram ${ }^{19}$ yields a spectrum which is the true spectrum multiplied by $\exp (i k \epsilon)$, where $\epsilon$ is the off-center error. Since both the on- and off-axis interferograms have the same off-center sampling error, this complex term cancels in the ratio, leaving the true relative field.

Finally, we use the fact that the aperture plane electric field at a given $k$ is the 2-D spatial Fourier transform of $E_{f f}\left(\phi_{x}, \phi_{y}, k\right)$. With $x_{a p}$ and $y_{a p}$ as linear coordinates in the aperture plane and the usual small angle approximation, the aperture plane field is, to within a constant of proportionality,

$$
\begin{aligned}
E_{a p}\left(x_{a p}, y_{a p}, k\right)=\int_{-\infty}^{\infty} \int_{-\infty}^{\infty} & r\left(\phi_{x}, \phi_{y}, k\right) \\
& \quad \times \exp \left[i k\left(x_{a p} \phi_{x}+y_{a p} \phi_{y}\right)\right] k^{2} d \phi_{x} d \phi_{y} .
\end{aligned}
$$

The spectral and spatial Fourier transforms discussed separately until now can in fact be combined into a single 3-D Fourier transform. Redefining the spatial coordinates on the dish to be $x=k x_{a p}$ and $y=k y_{a p}$, the complete expression for the aperture plane electric field as a function of location and wavenumber is then

$$
\begin{aligned}
E_{a p}(x, y, k)=\frac{k^{2}}{S(k)} \int_{-\infty}^{\infty} \int_{-\infty}^{\infty} \int_{-\infty}^{\infty} I_{m}\left(\phi_{x}, \phi_{y}, \delta\right) \\
\quad \times \exp \left[i\left(x \phi_{x}+y \phi_{y}+k \delta\right)\right] d \delta d \phi_{x} d \phi_{y} .
\end{aligned}
$$

\section{B. Measurement Procedure}

The complete dish measurement procedure is then as follows: The rotatable mirror $M 2$ is first set to a given angle, and $M 1$ is scanned, generating a crosspower interferogram which is sampled by a power detector. Because this interferogram contains flux from both the astronomical source and the atmosphere, the telescope is next shifted well off-source (about 6 arc minutes in azimuth in practice), and, with $M 2$ still in the same orientation, an off-source sky emission interferogram is obtained, and subtracted from the onsource interferogram. After mirror $M 2$ is set to a new position, the procedure is repeated, until $M 2$ has mapped out a complete $N \times N$ grid of far field positions. The angular spacing of this grid, $\Delta \phi_{s}$, is $\leqslant \lambda /$ $1.2 D$, to avoid spatial aliasing. ${ }^{24}$ Currently, $100 \mathrm{~min}$ are required to obtain a complete $21 \times 21$ point map.

Although it is possible to treat the data three dimensionally from the start, via Eq. (18) it is more convenient first to spectrally transform the interferograms, yielding a grid of cross-power far field spectra. After normalizing by the on-axis source spectrum, transforms to the aperture plane are then carried out at each 
$k$. The phase of the aperture plane field is next corrected for pointing, focus, and secondary centering errors by mathematically fitting and removing appropriate terms ${ }^{25}$ from the phase. The resultant phase map is then converted into the geometric deviations normal to the ideal surface. Finally, the surface error maps obtained for each spectral channel are averaged together to improve the SNR. In practice, a total bandwidth of about half of an octave is most useful because a broader band suffers from low spatial resolution at low frequencies and spatial aliasing at high frequencies.

\section{Resolution Considerations}

Although the effect of a finite bandwidth on the far field measurements was touched upon earlier, we now consider how the bandwidth affects the inverted aperture plane data. Since the far field is sampled on a discrete $N \times N$ grid, the aperture plane data will also consist of an $N \times N$ rectangular grid of data points. However, because the transform to the aperture plane requires multiplication by $k$-dependent terms, the wavelength independent far field observing grid is transformed to an aperture plane data grid with wavelength dependent spacing $g$ given $b^{5,24}$

$$
g=\frac{\lambda}{N \Delta \phi_{s}} \text {. }
$$

As we now show in detail, this implies that the effect of a finite bandwidth is to smear the aperture plane data in the radial direction.

We begin by modifying the theory to account for the limited scan length and far field sampling which must be used in practice. The spectral resolution is defined both by the maximum path difference reached by displacing mirror $M 1$, and by the numerical apodization applied to the data. ${ }^{19}$ Accounting for both of these effects with an apodizing function $a(\delta)$ which is zero beyond $\pm L$, and using the subscript $m$ to refer to the actually measured quantities, we have

$$
r_{m}\left(\phi_{x}, \phi_{y}, k\right)=\frac{1}{S(k)} \int_{-\infty}^{\infty} I_{m}\left(\phi_{x}, \phi_{y}, \delta\right) a(\delta) \exp (i k \delta) d \delta,
$$

or, in terms of the usual convolution integral, ${ }^{5}$

$$
r_{m}\left(\phi_{x}, \phi_{y}, k\right)=\int_{-\infty}^{\infty} r\left(\phi_{x}, \phi_{y}, k^{\prime}\right) A\left(k-k^{\prime}\right) d k^{\prime},
$$

where $A(k)$, the Fourier transform of $a(\delta)$, is the instrumental resolution function. As usual, the effect of a limited scan length is to convolve the actual quantity with the resolution function. ${ }^{19}$ (The effect of sampling $I_{m}$ at a discrete number of points has been considered elsewhere, ${ }^{5,19}$ and so is not addressed here; the interferogram need only be sampled finely enough so that no aliasing occurs.)

Inserting the convolution integral [Eq. (20)] for $r_{m}$ into Eq. (17) for the aperture plane field, and also including a spatial windowing function $w\left(\phi_{x}, \phi_{y}\right)$ which is zero outside the observed grid of points, to cut off the angular integrals, the measured aperture plane field is

$$
\begin{aligned}
E_{a p, m}\left(x_{a p}, y_{a p}, k\right) & =\int_{-\infty}^{\infty} \int_{-\infty}^{\infty}\left[\int_{-\infty}^{\infty} r\left(\phi_{x}, \phi_{y}, k^{\prime}\right) A\left(k-k^{\prime}\right) d k^{\prime}\right] \\
& \times w\left(\phi_{x}, \phi_{y}\right) \exp \left[i k\left(x_{a p} \phi_{x}+y_{a p} \phi_{y}\right)\right] k^{2} d \phi_{x} d \phi_{y} .
\end{aligned}
$$

Reversing the order of the integrations,

$$
\begin{aligned}
E_{a p, m}\left(x_{a p}, y_{a p}, k\right) & =\int_{-\infty}^{\infty} d k^{\prime} A\left(k-k^{\prime}\right) \int_{-\infty}^{\infty} \int_{-\infty}^{\infty} r\left(\phi_{x}, \phi_{y}, k^{\prime}\right) \\
& \times w\left(\phi_{x}, \phi_{y}\right) \exp \left[i k\left(x_{a p} \phi_{x}+y_{a p} \phi_{y}\right)\right] k^{2} d \phi_{x} d \phi_{y} .
\end{aligned}
$$

The inner two integrals are almost, but not quite, the standard Fourier transform to the aperture plane. Because of the outer convolution integral, function $r$ depends on $k^{\prime}$, but the exponent on $k$, and so, the phases contributing to the field at $\left(x_{a p}, y_{a p}\right)$ at wavenumber $k^{\prime}$, will not be summed properly. However, the phases will add up correctly if for a given $k^{\prime}$ we consider instead the point at

$$
\left(x_{a p}^{\prime}, y_{a p}^{\prime}\right)=\frac{k}{k^{\prime}}\left(x_{a p}, y_{a p}\right) \text {, }
$$

which is offset radially from $\left(x_{a p}, y_{a p}\right)$. Since, for high spectral resolution, $A\left(k-k^{\prime}\right)$ is large only for $k \approx k^{\prime}$, this spatial offset is small compared to a spatial resolution element. Making this coordinate substitution in the exponent then gives

$$
\begin{aligned}
& E_{a p, m}\left(x_{a p}, y_{a p}, k\right)=\int_{-\infty}^{\infty} d k^{\prime} A\left(k-k^{\prime}\right)\left(\frac{k}{k^{\prime}}\right)^{2} \int_{-\infty}^{\infty} \int_{-\infty}^{\infty} \\
& \quad \times r\left(\phi_{x}, \phi_{y}, k^{\prime}\right) w\left(\phi_{x}, \phi_{y}\right) \exp \left[i k^{\prime}\left(x_{a p}^{\prime} \phi_{x}+y_{a p}^{\prime} \phi_{y}\right)\right] k^{\prime 2} d \phi_{x} d \phi_{y} .
\end{aligned}
$$

The 2-D transform to the $\left(x_{a p}^{\prime}, y_{a p}^{\prime}\right)$ plane can now be done. Because it is the product of $r$ and $w$ which is being transformed, the result is again a convolution, giving

$$
\begin{aligned}
& E_{a p, m}\left(x_{a p}, y_{a p}, k\right)=\int_{-\infty}^{\infty} d k^{\prime} A^{\prime}\left(k-k^{\prime}\right) \int_{-\infty}^{\infty} \int_{-\infty}^{\infty} \\
& \times E_{a p}\left(x_{a p}^{\prime \prime}, y_{a p}^{\prime \prime}, k^{\prime}\right) W\left(x_{a p}^{\prime}-x_{a p}^{\prime \prime}, y_{a p}^{\prime}-y_{a p}^{\prime \prime}, k^{\prime}\right) d x_{a p}^{\prime \prime} d y_{a p}^{\prime \prime} .
\end{aligned}
$$

Here $A^{\prime}\left(k-k^{\prime}\right)=A\left(k-k^{\prime}\right)\left(k / k^{\prime}\right)^{2}$ is a slightly modified spectral resolution function, $W\left(x_{a p}^{\prime}, y_{a p}^{\prime}, k^{\prime}\right)$ is the Fourier transform of the windowing function $w\left(\phi_{x}, \phi_{y}\right)$ (a function of $k^{\prime}$ now), and the double-primed coordinates $\left(x_{a p}^{\prime \prime}, y_{a p}^{\prime \prime}\right)$ are dummy variables in the $\left(x_{a p}^{\prime}, y_{a p}^{\prime}\right)$ plane. In this equation, both $A$ and $W$ play the role of spatial resolution functions. To see this explicitly, we examine two limiting cases.

First, we assume monochromatic radiation and set $A^{\prime}\left(k-k^{\prime}\right)=\delta\left(k-k^{\prime}\right)$, the Dirac delta function. ${ }^{5}$ This implies that $\left(x_{a p}^{\prime}, y_{a p}^{\prime}\right)=\left(x_{a p}, y_{a p}\right)$ and yields

$$
\begin{aligned}
E_{a p, m}\left(x_{a p}, y_{a p}, k\right)=\int_{-\infty}^{\infty} & \int_{-\infty}^{\infty} E_{a p}\left(x_{a p}^{\prime \prime}, y_{a p}^{\prime \prime}, k\right) \\
& \times W\left(x_{a p}-x_{a p}^{\prime \prime}, y_{a p}-y_{a p}^{\prime \prime}, k\right) d x_{a p}^{\prime \prime} d y_{a p}^{\prime \prime} .
\end{aligned}
$$

This convolution of the true field $E_{a p}$ with $W$ is the usual result for a limited range of far field data ${ }^{5,24}$ and identifies $W$ as the spatial resolution function on the dish.

In the opposite limit, we assume instead that the far field has been measured arbitrarily far off-axis and set 
$W\left(x_{a p}^{\prime}, y_{a p}^{\prime}, k^{\prime}\right)=\delta\left(x_{a p}^{\prime}, y_{a p}^{\prime}, k^{\prime}\right)$, the 2-D spatial delta function. Equation (26) now gives

$$
E_{a p, m}\left(x_{a p}, y_{a p}, k\right)=\int_{-\infty}^{\infty} d k^{\prime} E_{a p}\left(x_{a p}^{\prime}, y_{a p}^{\prime}, k^{\prime}\right) A^{\prime}\left(k-k^{\prime}\right),
$$

or, more explicitly,

$$
E_{a p, m}\left(x_{a p}, y_{a p}, k\right)=\int_{-\infty}^{\infty} d k^{\prime} E_{a p}\left(\frac{k}{k^{\prime}} x_{a p}, \frac{k}{k^{\prime}} y_{a p}, k^{\prime}\right) A^{\prime}\left(k-k^{\prime}\right) .
$$

For each $k^{\prime}$ which contributes to the spectral bandpass, the field from a different radial position enters into this convolution integral. Thus, in this case, the spectral resolution function $A^{\prime}(k)$ assumes the role of the spatial resolution function. Since Eq. (28) smears the data only in the radial direction, the shift in radius $\rho$ is given as a function of wavenumber by

$$
\Delta \rho=-\frac{k \rho}{k^{\prime 2}} \Delta k,
$$

or, since $k^{\prime} \approx k$,

$$
\frac{\Delta \rho}{\rho} \approx \frac{\Delta k}{k} \equiv \frac{1}{R} \text {. }
$$

The spatial resolution element $\Delta \rho$ is thus proportional to both the radius and the bandwidth.

In practice, the spectral resolution should be high enough so that $W$, and not $A$, determines the spatial resolution. For this to be the case, the bandwidth determined spatial resolution at the edge of the dish, $\Delta \rho_{\text {edge }}(=D / 2 R)$, should be much smaller than the spacing of the aperture plane data grid given by Eq. (19). Taking the ratio $\left(\Delta \rho_{\text {edge }} / g\right)$, and solving for the spectral resolution, we get

$$
R=\frac{N}{2}\left(\frac{\Delta \phi_{s}}{\lambda / D}\right)\left(\frac{g}{\Delta \rho_{\text {edge }}}\right) \text {. }
$$

Since the first term in parentheses should be $\approx 1 / 1.2$ to avoid aliasing in the aperture plane ${ }^{24}$ and the second term should be $\gtrsim 2$ to avoid having the bandpass determine the spatial resolution, we require $R \gtrsim N / 1.2$. This agrees well with our earlier estimate (Sec. II.B), if we arbitrarily set $n=N / 2$. However, the present result is much more rigorously established.

\section{Instrumentation}

We now turn to the description of the actual interferometer constructed for use at the CSO. The necessary optical components are quite few, being two numerically milled off-axis paraboloids, two flat glass end mirrors on gimbal mounts, a $45^{\circ}$ flat glass mirror to send the output beam to a more convenient location, and a Mylar beam splitter. The paraboloids must be very much oversized because of beam walkoff induced by rotation of M2. A polarizing Martin-Pupplett interferometer $^{26}$ cannot be used, because mirror $M 2$ must be capable of steering its beam in two orthogonal directions, while the rooftop reflectors used in polarizing interferometers can steer the beam in one direction only. Thus, although a polarizing interferometer would result in a higher SNR, ${ }^{26}$ this choice is ruled out.

The effects of atmospheric fluctuations can be

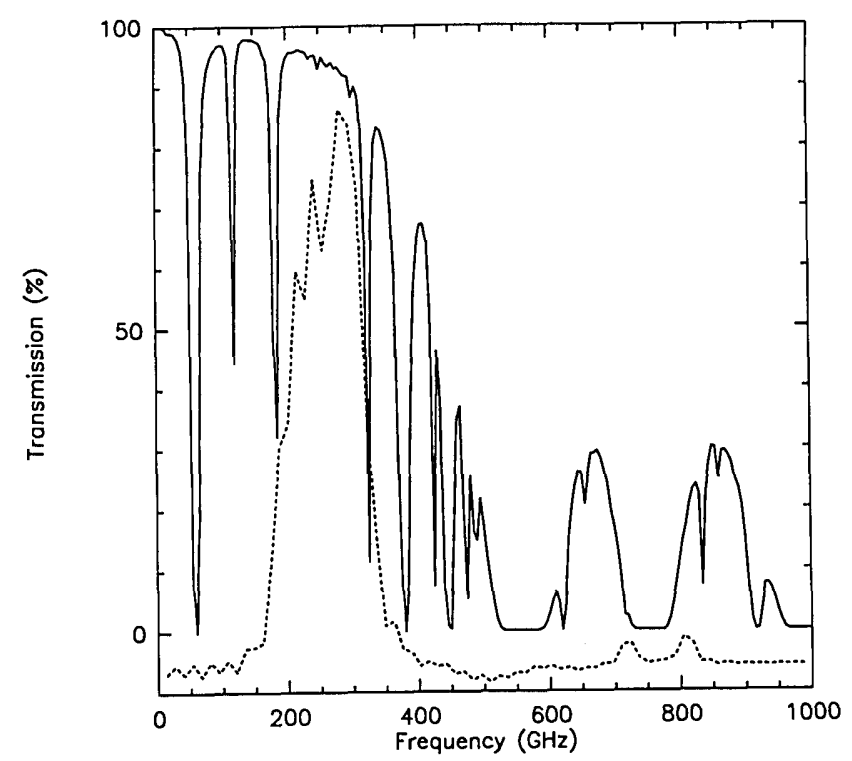

Fig. 2. Solid curve, calculated CSO zenith atmospheric transmission for $1.5-\mathrm{mm}$ precipitable water vapor; dashed curve, measured transmission of the filter designed to match the $200-300-\mathrm{GHz}$ atmospheric window. The vertical scaling of this curve is arbitrary.

avoided by scanning the FTS rapidly. ${ }^{20,26}$ With the mirror moving at $8 \mathrm{~mm} / \mathrm{s}$, a signal at $\lambda=1 \mathrm{~mm}$ will be modulated at $16 \mathrm{~Hz}$, well above the frequencies of sky fluctuations and detector $1 / f$ noise. A simple de motor and lead screw attached to a commercial precision translation stage have been found to give adequately rapid and linear travel at millimeter wavelengths, the only difficulty being the suppression of vibrations. The total usable travel of $\sim 35 \mathrm{~mm}$ limits the spectral resolution to $5 \mathrm{GHz}$. The position of the translating mirror is determined with a commercial magnetic transducer, which was calibrated with a $\mathrm{He}-\mathrm{Ne}$ laser interferometer. The other interferometer mirror, $M 2$, is rotated into position with two stepper motors.

The detector used is a Si bolometer ${ }^{27}$ maintained at a temperature of $0.32 \mathrm{~K}$ in a cryogenic Dewar. The bolometer is mounted in an integrating cavity behind a Winston cone concentrator. ${ }^{28}$ The entrance aperture of the Winston cone is located in the output focal plane of the interferometer, with a diameter corresponding to $30 \mathrm{sec}$ of arc on the sky. For comparison, $1.22(\lambda / D)$ is $24 \mathrm{sec}$ of arc at $\lambda=1 \mathrm{~mm}$. This small but finite detector size does not affect the results greatly, as explained in Appendix A.

The bandwidth of the radiation reaching the detector is determined by cold filters inside the Dewar. The band is limited on the low frequency side by the waveguidelike cutoff of the output end of the Winston cone, ${ }^{28}$ and on the high frequency side by a $4.2 \mathrm{~K}$ low pass metal mesh filter. ${ }^{29}$ The first-order interference maximum of the Mylar beam splitter also aids in defining the passband. ${ }^{11}$ To reject far infrared through optical radiation, two blocking filters are used: a 14mil thick sheet of fluorogold ${ }^{30}$ located on the $77-\mathrm{K}$ radiation shield, and a 25 -mil thick piece of quartz ${ }^{31}$ at 
$4.2 \mathrm{~K}$, the latter being antireflection coated with black polyethylene.

The actual bandpass used was chosen to match the 200-300-GHz atmospheric transmission window evident on the theoretical curve ${ }^{32}$ shown in Fig. 2. To this end, a $325-\mathrm{GHz}$ low pass filter and a $175-\mu \mathrm{m}$ thick Mylar beam splitter were employed. As Fig. 2 shows, the instrumental transmission measured with the interferometer itself is a good match for the atmospheric window.

\section{Experimental Results}

\section{A. Cross-Power Interferograms and Spectra}

The first test of the interferometer was an examination of the cross-power interferograms and specta obtained on a distant source. Since the only far field sources strong enough are planets, which have finite extent, the effect of a finite source size is discussed in Appendix B. There it is shown that, as long as the angular diameter of the source is somewhat smaller than the angular diameter of the first dark ring of the telescope's Airy pattern, the loss of coherence is not a significant problem. The sources actually used were Venus, Mars, and Jupiter, which at the time of the measurements had sizes of 28,23 , and $44.5 \mathrm{sec}$ of arc, respectively. Based on the coherence arguments, there is no problem with the first two, but there is a significant loss of coherence near the edge of the dish for Jupiter. In the final aperture plane maps, this translates into a lower SNR near the edge of the dish for Jupiter.

Figure 3(a) shows cross-power interferograms obtained on Mars on 10 Sept. 1988 for three orientations of M2: the top one with $M 2$ aligned on-axis, and the middle and bottom ones with $M 2$ aligned to send its beam to the sky off-axis by 34 and 54 sec of arc, respectively. The atmospheric emission has been subtracted via an off-source measurement. The cross-power spectra obtained by Fourier transforming these three interferograms are shown in Fig. 3(b).

The on-axis interferogram is quite symmetrical and normal and produces primarily a real, positive spectrum, with a small imaginary component introduced by noise and pointing errors. The bottom interferogram is also reasonably symmetric, but much weaker, and inverted; the central finge is now negative, and the entire fringe pattern follows suit, resembling an upside down version of the top interferogram. As a consequence, the spectrum at this position is also inverted, being entirely negative (with again a small imaginary component). Since the spectrum is proportional to the off-axis field, evidently the far field at this position is opposite in direction to the field at the center of the pattern for all frequencies in the band. Such a flip in field direction is just what occurs in an Airy pattern; the electric field direction in the first bright ring is opposite that in the central maximum. ${ }^{12}$ Thus, the interferometer has apparently measured the change in electric field direction correctly. (In general, phase
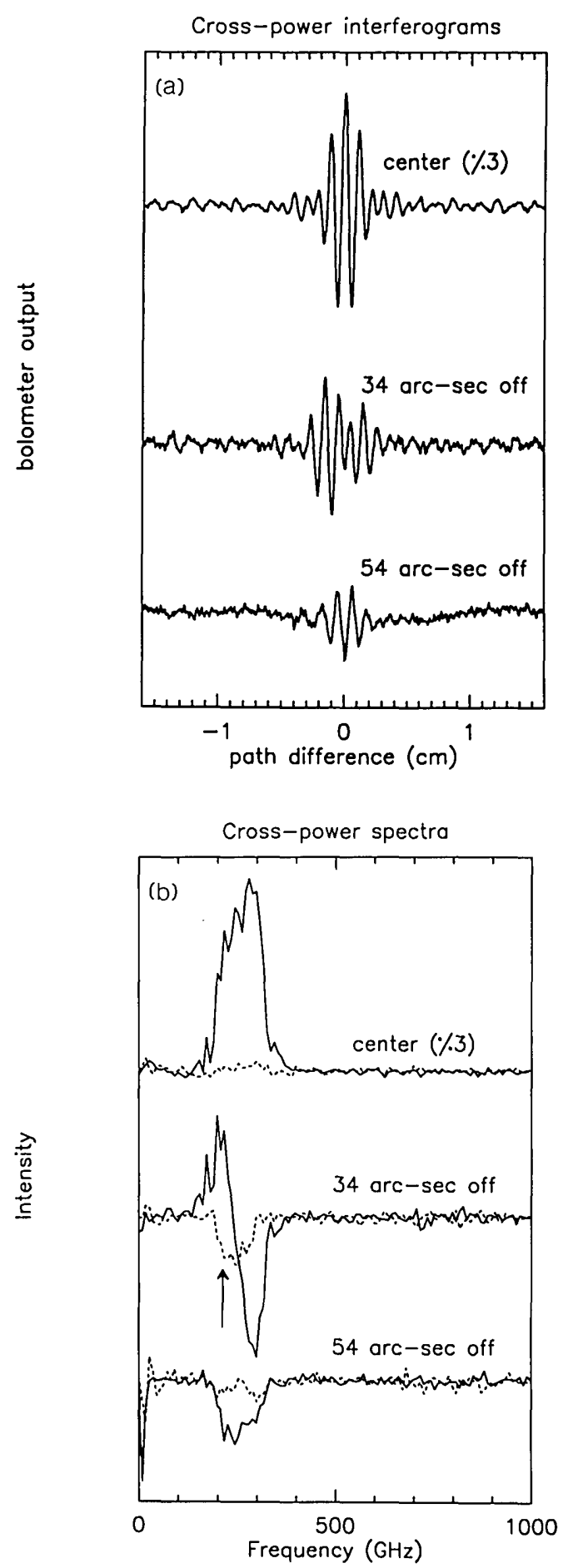

Fig. 3. (a) Cross-power interferograms measured on Mars for three orientations of mirror M2. (b) Corresponding cross-power spectra. The solid and dashed lines give the real and imaginary parts of the Fourier transform, respectively. The arrow indicates the frequency for which the first dark Airy ring is $34 \mathrm{sec}$ of arc off-axis.

shifts other than a simple $180^{\circ}$ reversal will introduce imaginary components into the cross-power spectra.)

The spectrum $34 \mathrm{sec}$ of arc off-axis [Fig. 3(b), middle] confirms this. At this location, the high frequencies have flipped sign, but the low frequencies have not. Theoretically, the first dark Airy ring produced 


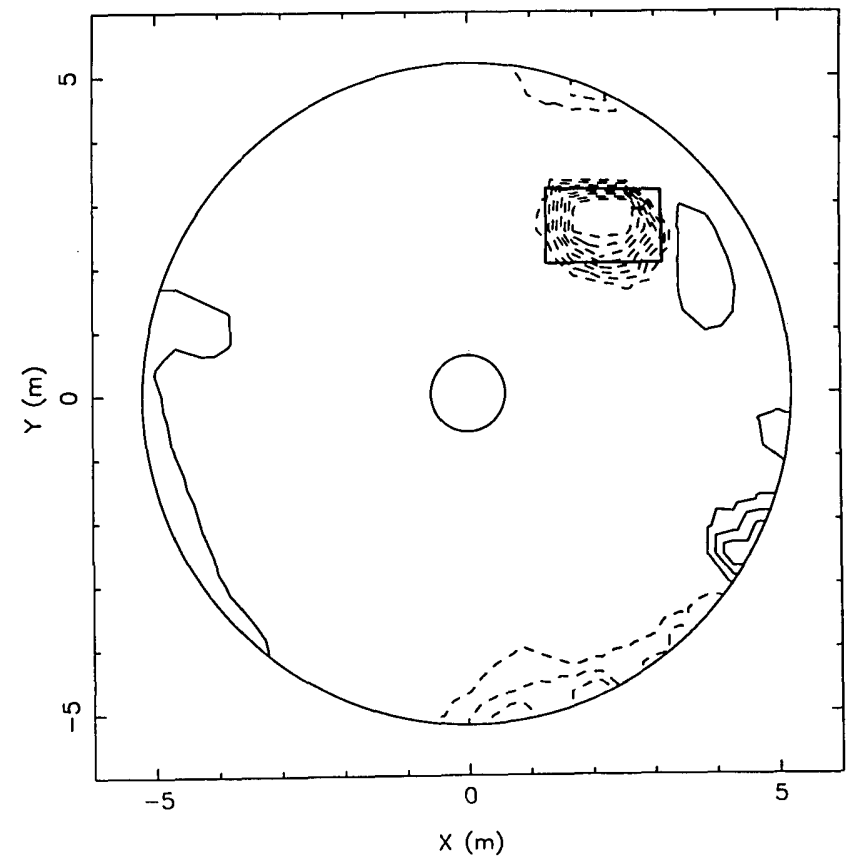

Fig. 4. Measured difference map between two telescope aperture plane phase maps, one made with and one without a $250-\mu \mathrm{m}$ thick piece of Mylar located at the position of the rectangle in the upper right quadrant of the dish. The contour levels are $\pm 25 \mu \mathrm{m}$, the grid size $15 \times 15$, and the spacing of the data points $0.85 \times 0.85 \mathrm{~m}$. The small centered circle shows the approximate central obscuration.

by a 10.4-m diam aperture lies $34 \mathrm{sec}$ of arc off-axis for a frequency of $213.5 \mathrm{GHz}$, indicated in Fig. 3(b) by the arrow. Since this frequency is quite close to the position at which the spectrum flips sign, evidently at this position the low frequencies are still within the boundaries of the central maximum of the Airy pattern, while the higher frequencies lie in the first bright ring. As a result of this loss of phase uniformity across the band, there is apparently no longer any path difference setting of the interferometer where all the frequency components are in phase, and consequently, this interferogram is not symmetric.

The bottom interferogram and spectrum also show the effect of atmospheric fluctuations: a wobbly baseline. However, since this variation is slow $(\approx 0.5 \mathrm{~Hz})$, it transforms to very low frequencies $(\approx 10 \mathrm{GHz})$, well out of the range of interest. This allows observation even in nonideal conditions.

\section{B. Initial Holographic Tests}

The first test of the interferometer's dish measurement capabilities was to introduce a known phase shift on a small section of the surface, to determine if it could be detected. To this end, a rectangular sheet of $80-\times 50$-in. Mylar (index of refraction = 1.75), $0.01 \mathrm{in}$. thick, was taped to the primary surface, and the aperture plane phase distribution was measured with the interferometer on 9 Sept. 1988. To directly see its effect, this phase map was subtracted from a phase map made at similar zenith angles the previous day.

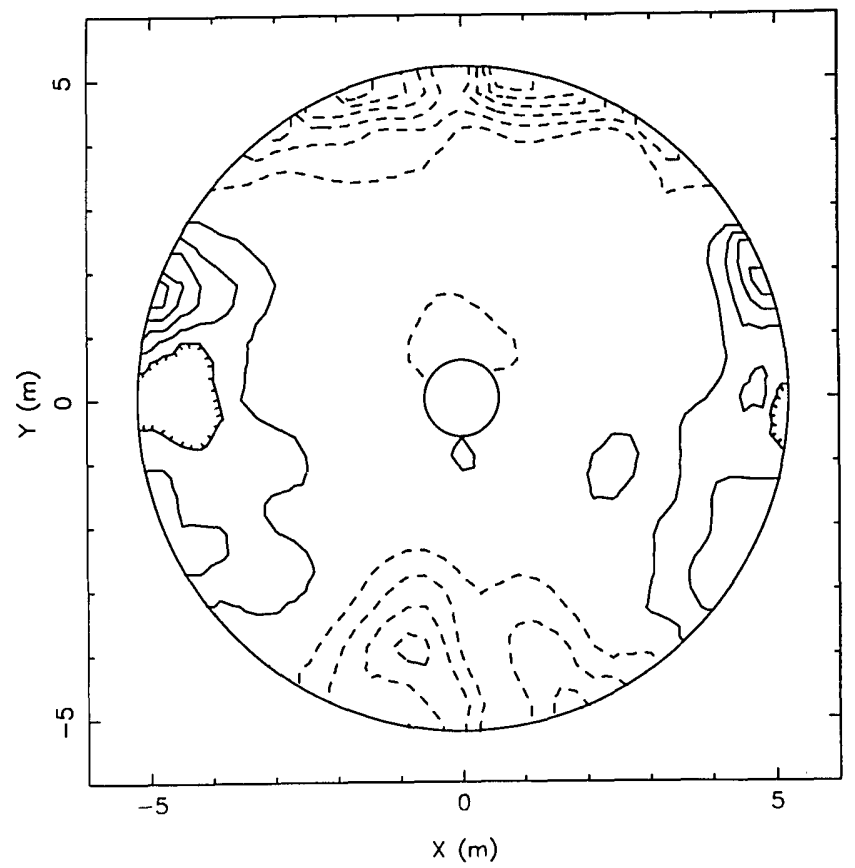

Fig. 5. Measured differences between two $21 \times 21$ point dish surface maps made over similar zenith angle ranges $\left(10^{\circ} \pm 7^{\circ}\right.$ vs $12^{\circ} \pm$ $\left.8^{\circ}\right)$. For this and all succeeding figures, the solid contours show 10 $\mu \mathrm{m}$ increments in height above the ideal surface, and the dashed contours $10-\mu \mathrm{m}$ decrements below the ideal surface.

The phase difference map obtained by selecting the $270 \mathrm{GHz}$ data from the spectra is shown in Fig. 4. (The orientation of this, and all subsequent maps presented, is the view of the dish obtained when tipping the telescope to the horizon and looking at the front surface of the primary mirror.) Although the resultant spatial resolution is somewhat limited, the phase shift due to the Mylar clearly shows up in the correct location, with approximately the correct amplitude, thus verifying the basic measurement technique. The figure also gives some indication of the reproducibility of the technique, as most of the remainder of the difference map is blank.

The reproducibility of the surface error maps is, however, better addressed with data obtained about a year later, after various improvements to the optical and data-taking systems. Figure 5 shows the difference between two surface deviation maps obtained on Jupiter near zenith on 15 and 18 Nov. 1989. The grid size and sampling on the dish are $21 \times 21$ and $\sim 0.6 \mathrm{~m}$, respectively, and the central frequency and resolution, 290 and $10 \mathrm{GHz}$, respectively. The solid (dashed) contours give the differences of the surface shapes measured on the two occasions, in increments of $10 \mu \mathrm{m}$ above (below) the ideal paraboloidal surface. Except for one small region near the bottom of the map, the errors within the central 4-m radius show a repeatability of $<10 \mu \mathrm{m}$. In the outer meter, the reproducibility gets worse, likely due to the falloff of the illumination function seen in the same region (next section), and to the loss of coherence at the edge of the aperture due to 

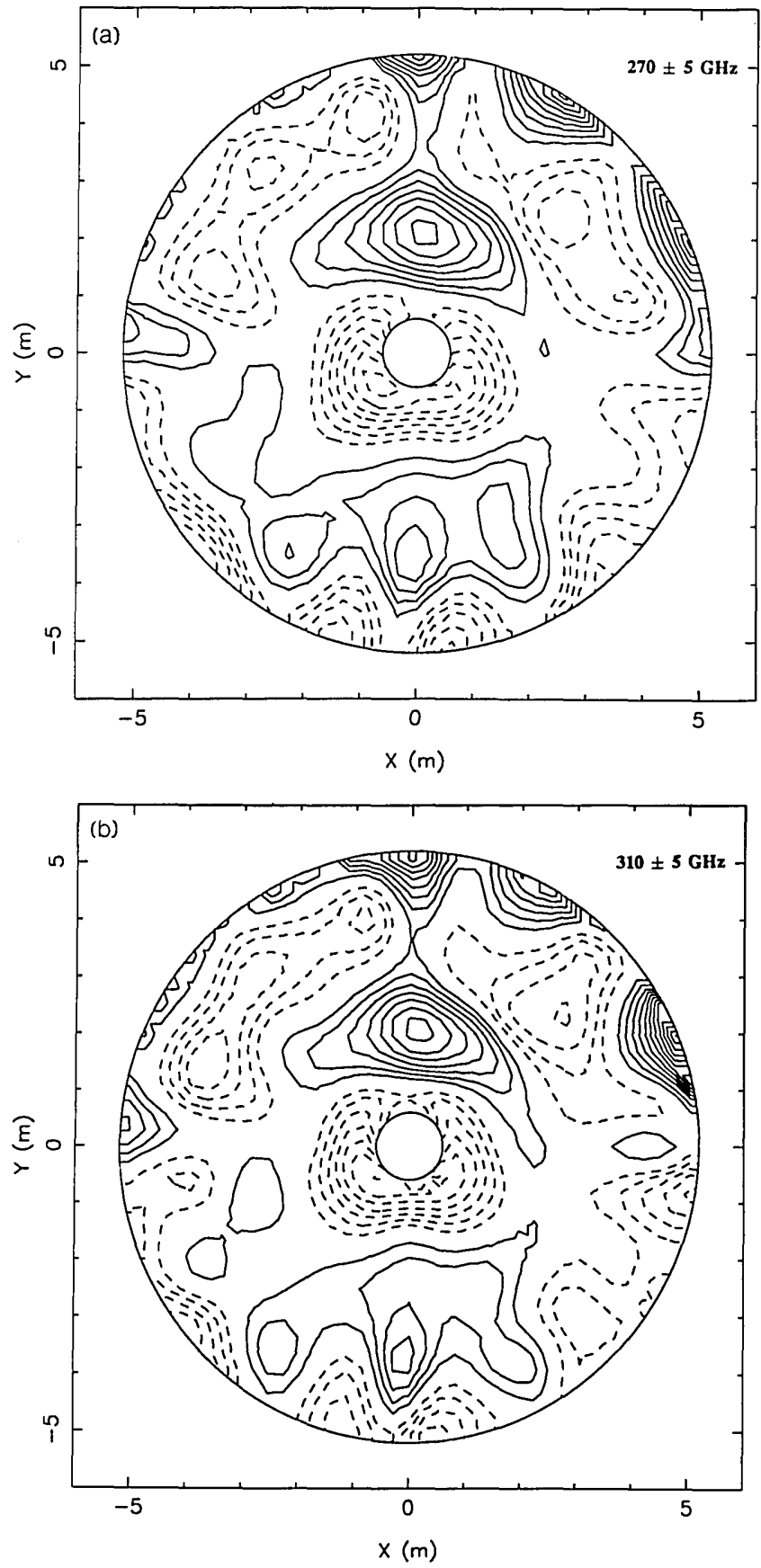

the extended source (Appendix B). It is also likely that the dish itself is not as stable at the edges as at the center. The rms deviation of the difference map is 13 $\mu \mathrm{m}$ when weighted by the dish illumination function. Consequently, the rms deviation of either map from the mean is $\sim 9 \mu \mathrm{m}$, or $\lambda / 115$. With a smaller source and a better illumination of the dish edge, the reproducibility would presumably be better still.

As a final check, aperture plane maps obtained simultaneously at widely separated frequencies were compared. Figures 6(a) and (b) show two maps measured on Jupiter near zenith, both with $10-\mathrm{GHz}$ bandwidth, but centered at 270 and $310 \mathrm{GHz}$, respectively.

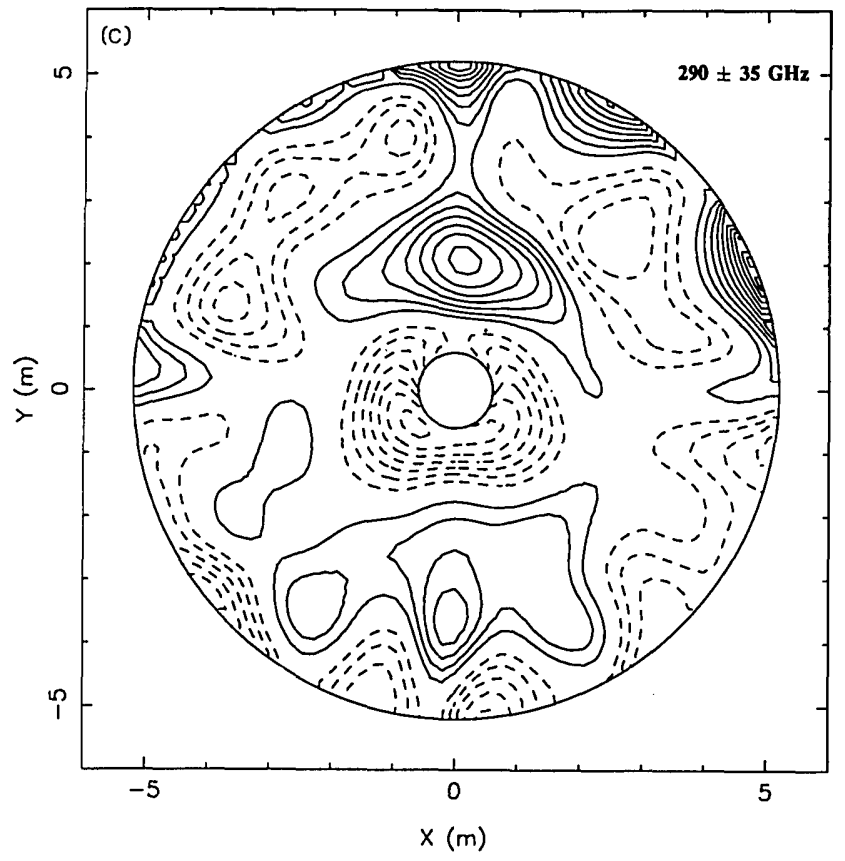

Fig. 6. The $21 \times 21$ point dish surface error maps made with Jupiter in the zenith angle range of $10^{\circ} \pm 7^{\circ}$ at the frequencies indicated in the upper right-hand corners of the maps.

A summed map made by integrating the data from 255 to $325 \mathrm{GHz}$ is shown in Fig. 6(c). There is clearly little change in the maps with frequency. The absolute surface error near zenith determined from these maps is $28 \mu \mathrm{m} \mathrm{rms}$.

Because any detected aperture plane phase shift could arise from an error on either the primary or secondary surfaces, aperture plane phase maps were obtained for two orientations of the secondary mirror, differing from each other by a rotation of $120^{\circ}$ about the optical axis. The resultant maps were quite similar. Likewise, after careful alignment, rotation of the interferometer by $90^{\circ}$ about the telescope axis was also 

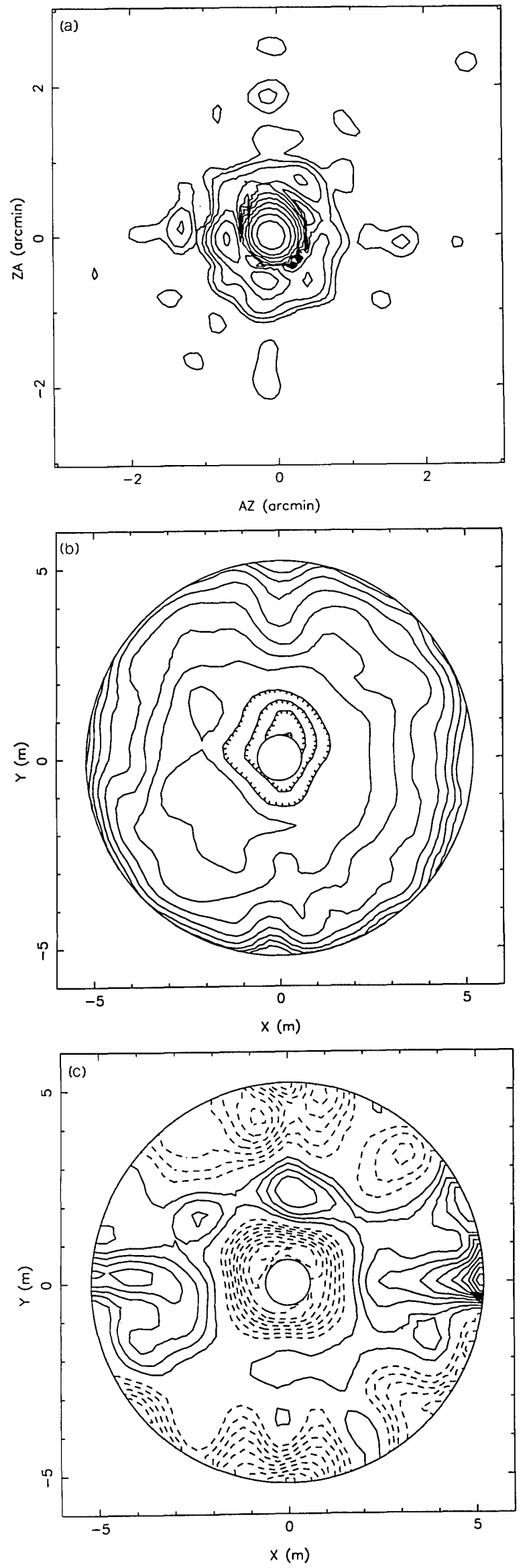
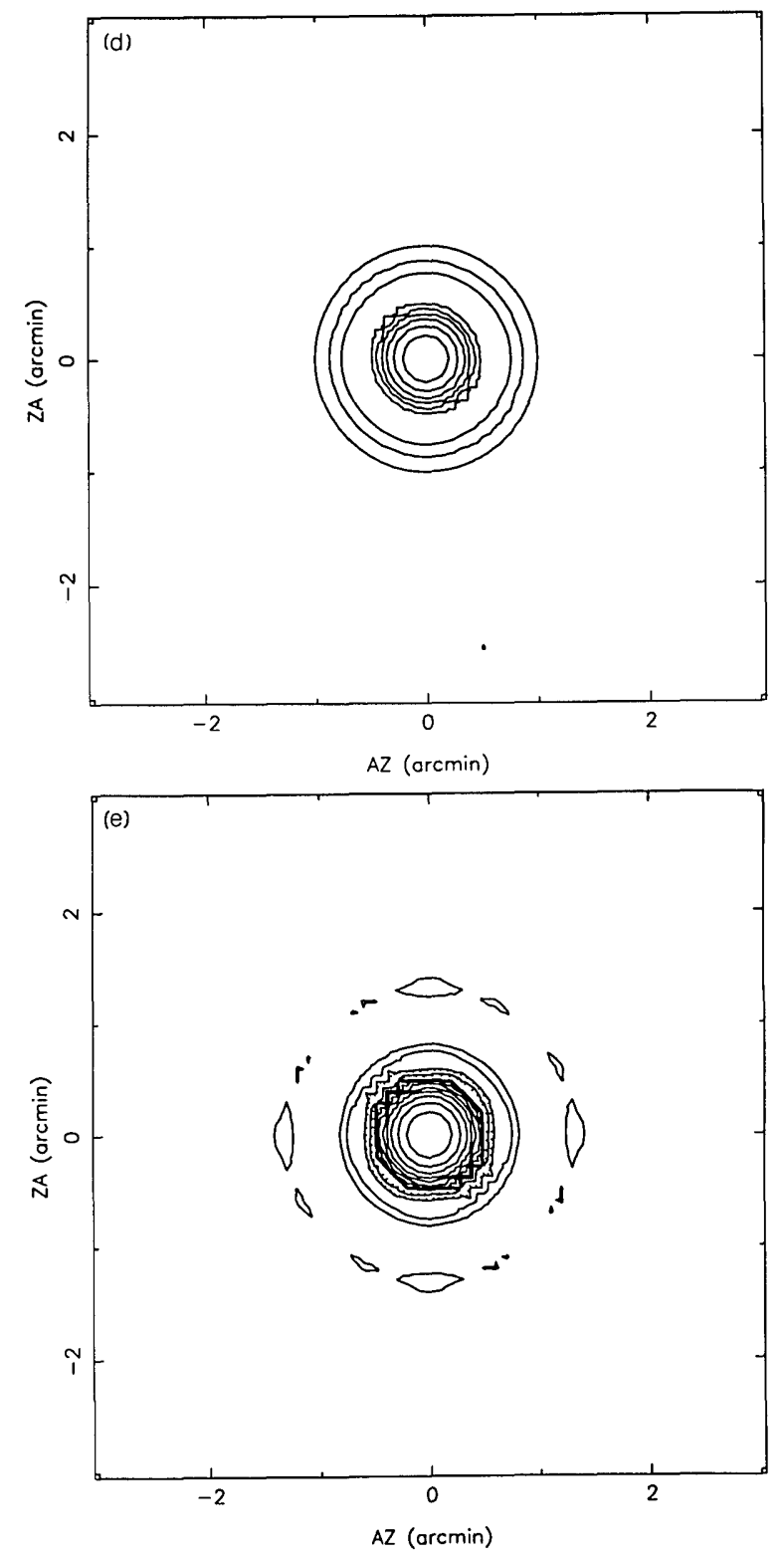

Fig. 7. (a) Far field power map made in the zenith angle range of $65^{\circ} \pm 10^{\circ}$ on Venus. The contour interval is $3 \mathrm{~dB}$ [also for (d) and (e)]. (b) Aperture plane illumination with 2-dB contours. (c) Aperture plane phase with $\pm 10-\mu \mathrm{m}$ contours. (d) Calculated far field power pattern for a 10.4-m dish with a $70-\mu \mathrm{m}$ dip over the central 3 m. (e) Calculated pattern with uniform phase over the entire dish, but with a central obscuration of 1.2-m diameter. 
found to leave the measured phases unaffected. Thus, with the possible exception of some circularly symmetric errors, the phase deviations detected should all be due to the primary mirror surface.

\section{Detailed Far Field and Aperture Plane Maps}

We now discuss one set of dish measurements in more detail. Figure 7 (a) presents a typical far field power map, made from a $21 \times 21$ grid of observations of Venus on 18 Nov, 1989. This map was measured over the zenith angle range of $65^{\circ} \pm 10^{\circ}$ and consists of 290 $\pm 5-\mathrm{GHz}$ data. The beam consists primarily of a welldefined central peak of $\sim 25$-sec of arc FWHM and a broader (80-sec of arc) plateau at the -15 to $-20-\mathrm{dB}$ level. Since the plateau is somewhat asymmetric, there may be some coma present. There is also evidence at the -24-dB level of linear spurs along the coordinate axes, most likely due to diffraction off the four feed legs, which are located in the horizontal and vertical planes.

Figure 7(b) shows the aperture plane power map produced from these far field data. This aperture plane distribution, which results mainly from the illumination pattern of the bolometer on the primary mirror, is a bit off-center, suggesting that the Dewar axis is not aligned perfectly with the optical axis. Nevertheless, the illumination is reasonably uniform over most of the dish and falls off steeply only in the outermost meter or so. The hole in the center of the primary is evident in the figure, being on average $7 \mathrm{~dB}$ down from the ring around it. Since the central hole is the size of one panel, the central depression indicates that panel sized errors are quite well resolved with a map of this size. Surprisingly, the contours curve inward from the edge along the $y$-axis, giving a hint of the blockage due to the vertical feed legs even with this low resolution. The horizontal feed legs are not evident. This relative strength is consistent with the far field power pattern [Fig. 7(a)] in which more power is scattered laterally than in zenith angle.

Figure 7(c) shows the surface deformations determined from this far field map. Although several features are noteworthy, only the central 3-m sized depression of $\sim 70-\mu \mathrm{m}$ depth is touched upon. This central depression is also seen in Fig. 6, but because its top portion is partially filled in by a strong peak of opposite sign located $\sim 2 \mathrm{~m}$ above the center of the dish, it appears to be shifted lower in those maps. Noting that the ratio of the dish diameter to the central depression diameter is $\sim 3$, similar to the ratio of the sizes of the plateau and main beam components in the far field map [Fig. 7(a)], it is reasonable to guess that the two are related. Therefore, an expected far field pattern was calculated for an aperture with a 70- $\mu \mathrm{m}$ depression in the central $3 \mathrm{~m}$, but which is otherwise perfect. The amplitude distribution was assumed Gaussian, with 15-dB edge illumination. As Fig. 7(d) shows, the calculated far field pattern exhibits a low level plateau around the main beam, of quite similar size and height to the plateau seen in Fig. 7(a). In contrast, simply including the appropriate central ob-
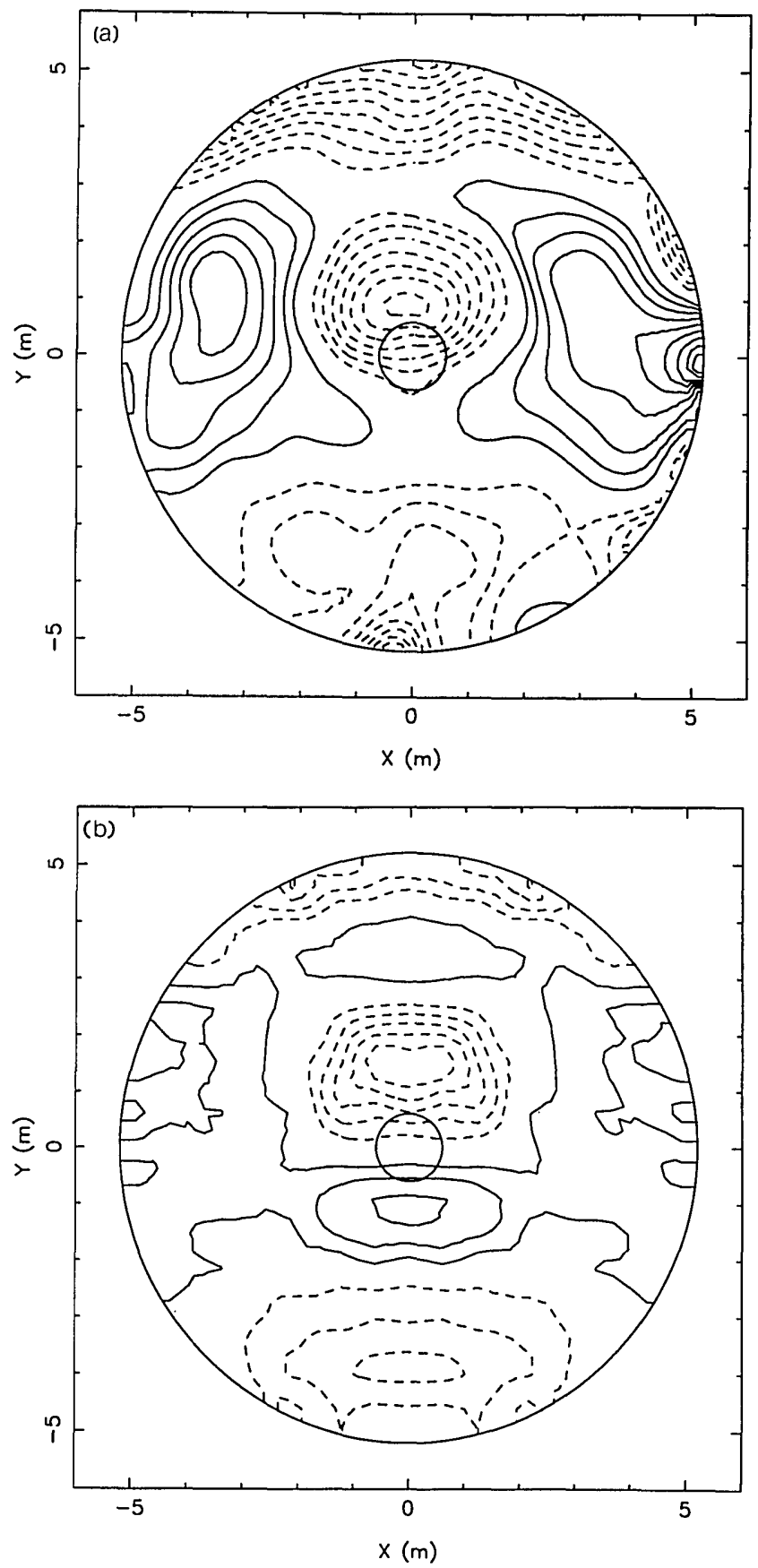

Fig. 8. (a) Measured telescope surface deformations incurred when tipping the telescope from $10^{\circ}$ zenith angle to $65^{\circ}$. Grid size $=21 \times$ 21 , and spacing of data points on the dish $=0.6 \mathrm{~m}$. Frequency $=290$ $\pm 35 \mathrm{GHz}$. (b) Theoretical predictions of the same. Contour intervals for both maps are $10 \mu \mathrm{m}$. Solid contours indicate an upward (toward the prime focus) displacement of the surface when tipping and dashed contours an opposite movement.

scuration $(1.2 \mathrm{~m})$ in the calculations does not reproduce the observed plateau [Fig. 7(e)]. Thus, this central phase error is clearly related to near-axis structure in the far field response, and its removal should help considerably in improving the telescope beam. Based on crude mechanical measurements, this error is now thought to lie on the secondary. 


\section{Elevation Dependence of the Dish Surface Shape}

Finally, in keeping with the initial goals, surface maps obtained at differing elevation angles were compared to see the deformations incurred when tipping the telescope. Figure 8(a) shows a map of the deviations normal to the surface which remain after subtracting the high elevation aperture plane map obtained on Jupiter (Fig. 6) from the low elevation Venus map of Fig. 7(c). The zenith angle ranges for the two maps are $65^{\circ} \pm 10^{\circ}$ and $10^{\circ} \pm 7^{\circ}$, respectively. The measured elevation dependent deformation shows a remarkably smooth and laterally symmetric pattern. The dominant effect appears to be approximately astigmatic, with an additional large dip located $\sim 1 \mathrm{~m}$ above the center of the dish. The rms deviation of this difference map is $28 \mu \mathrm{m}$.

If both planets were much smaller than the telescope beam, the fact that the two aperture plane maps were obtained on different planets would be irrelevant. However, as already mentioned, since Jupiter's size was not negligible at the time of the observations, its map is not very sensitive near the edge of the dish. Even so, the results are already most encouraging.

Figure $8(\mathrm{~b})$ shows a theoretical prediction ${ }^{33}$ of the dish deformation over the same zenith angle range. The agreement with theory is quite impressive in the overall shape of the deformations, especially if one compares the shape of the cuts along the $y$-axis. The depression $1 \mathrm{~m}$ above center is reproduced almost exactly and is apparently the location where the telescope support bears most of the weight of the telescope structure. ${ }^{34}$ The pulling on the surface due to the top feed leg is also apparent as a high spot along the $y$-axis between the two depressed regions. The only discrepancy between the measurements and theory is in the magnitude of several of the deformations; the measured deformations appear to be higher than predicted at the sides of the dish and also near the top. Given the uncertainties in the theoretical calculations at this point, ${ }^{34}$ the measured map is expected to be the more reliable of the two. In any case, it is clear that the new measurement technique presented here is fully capable of measuring the elevation dependent distortions of the reflector.

\section{Summary}

To optimize the surface figure of the primary mirror of the CSO, a new holographic measurement technique has been devised, which is based on the examination of two images of the primary with a shearing interferometer. With this technique of shearing holography, dish measurements can be obtained at the high frequencies for which the telescope is designed with the use of only a power detector and simple metal and glass opticsno heterodyne receiver or correlator is required. The use of a direct detector provides a substantial increase in sensitivity over corresponding heterodyne techniques. At the same time, the surface phase errors are a larger fraction of a wavelength at high frequency, and so the requirements on signal-to-noise ratio are re- duced. An additional benefit of using high frequencies is that the optical elements are large compared to a wavelength, so that there are no additional diffraction effects to deal with (the secondary diameter is $\sim 1000 \lambda$ at $\lambda=1 \mathrm{~mm}$ ).

Since the technique of shearing holography is inherently broadband in nature, broadband sources are a good match for the technique. This allows the use of astronomical continuum objects, such as the planets, as far field sources, bringing with it the advantage of being able to measure the deformation of the dish with changes in the zenith angle of the telescope.

The technique has been tested on the CSO and been found to work rather well. In particular, the reversal of field direction in successive rings of the Airy pattern has been measured, a known phase shift on the dish surface has been detected, the surface error maps have been found to be independent of the frequency selected (except for the expected decrease in resolution toward lower frequency), and a prominent feature of the telescope beam has been correlated with a large-scale surface error. However, most importantly, the zenith angle dependence of the primary reflector shape has been measured and has been found to agree remarkably well with theoretical calculations, thus providing a method for the near term improvement and eventual active control of the dish surface.

The authors wish to express their gratitude to $\mathrm{K}$. Young for single-handedly integrating the interferometer with the computer system at the CSO; W. Schaal, D. Vail, P. Schaffer, and B. Ellison for assistance in constructing the interferometer; J. B. Keene for assistance with the bolometer and filters; D. P. Woody for providing the OVRO holography software and the dish model calculations; J. H. Davis, D. Morris, J. H. Lacy, and again D. P. Woody for illuminating discussions; and A. Schinkel and the staff of the CSO for endless on-the-spot assistance. This work was supported by NSF grant AST88-15132.

\section{Appendix A. Extended Detector}

We now discuss the consequences of a nonpointlike detector. For simplicity, we assume that the exit aperture in the final focal plane corresponds to the actual detector surface, as in the near infrared case, and ignore the details of the Winston cone. For an extended detector, Eq. (14) for the measured interferogram is modified to

$$
\begin{aligned}
I_{m}\left(\phi_{x}, \phi_{y}, \delta\right) & =\int_{\Sigma} d \phi_{x}^{\prime} d \phi_{y}^{\prime} \int_{-\infty}^{\infty} T(k) f\left(\phi_{x}^{\prime}-\phi_{x}, \phi_{y}^{\prime}-\phi_{y}, k\right) \\
& \times E_{f f}^{*}\left(\phi_{x}^{\prime}-\phi_{x}, \phi_{y}^{\prime}-\phi_{y}, k\right) E_{f f}\left(\phi_{x}^{\prime}, \phi_{y}^{\prime}, k\right) \exp (-i k \delta) d k
\end{aligned}
$$

where $\Sigma$ represents the detector area, $f$ represents the responsivity of the detector as a function of position on its surface, and the detector geometry is described in terms of projected angles on the sky.

This integral represents a convolution of the desired quantity $E_{f f}$ with the product of the detector responsivity and the centered electric field pattern $E_{f f}^{*}$. Since both of these fall off toward the edge of the exit aper- 
ture, they can be combined into a single detector function. The convolution of the diffraction pattern with the detector function then implies that, in the transform to the aperture plane, the true aperture plane distribution is multiplied by the transform of the detector function. Considering the simplest case of a circular detector with uniform response across the exit aperture and neglecting the falloff of the centered term, the transform is an Airy pattern centered on the dish. Since this multiplies the aperture plane field, the primary effect of a finite-sized detector on the aperture plane data is a decrease in measurement sensitivity with radius.

To maintain sensitivity to the edge of the dish, the diameter $d_{z}$ of the first dark ring of this aperture plane Airy function must exceed the dish diameter $D$. Since

$$
d_{z}=2.44 \frac{\lambda}{\phi_{d}}
$$

where $\phi_{d}$ is the detector diameter, in units of its projected angular size on the sky, the detector diameter for which the first zero of the aperture plane Airy function is at the edge of the dish is

$$
\phi_{d}=2.44 \frac{\lambda}{D}
$$

which is equal to the diameter of the first dark ring in the ideal far field Airy pattern. Thus, in angular units, the detector diameter need only be somewhat less than the diameter of the telescope's first dark Airy ring (48 sec of arc at the CSO for $\lambda=1 \mathrm{~mm}$ ). In practice, the requirement is somewhat less stringent, because both contributions to the detector function fall off near the edge of the detector, implying a broader transform in the aperture plane.

\section{Appendix B. Extended Source}

The use of a planet as the source can be treated in a similar manner to the case of a finite-sized detector. On the other hand, it is also possible to analyze this situation with the aid of partial coherence theory. ${ }^{12}$ Here, rather than following the partially coherent radiation through the entire optical system, and then transforming back to the aperture plane, we take the more direct approach of simply examining the spatial coherence of the field produced by an extended source in the aperture plane of the telescope.

The van Cittert-Zernike theorem ${ }^{12}$ says that the complex coherence between the fields at two points in the aperture plane of the telescope, due to an incoherent and quasimonochromatic source of small extent in the telescope's far field, is given by the Fourier transform of the source intensity distribution. Assuming that the planet can be modeled as a uniform circular source of angular diameter $\alpha$, the magnitude of the coherence between the fields at the center of the telescope aperture and a point at radius $\rho$ is given ${ }^{12}$ by the magnitude of $\left[2 J_{1}(v)\right] / v$, where $J_{1}$ is the Bessel function of first kind and order, and $v=k_{o} \alpha \rho / 2$, with $k_{o}$ the average $k$ in the band. The edge of the dish is completely incoherent with the center at $v=1.22 \pi$ or

$$
\alpha=2.44 \frac{\lambda}{D}
$$

This is identical to Eq. (35), if we replace the detector diameter by the planetary diameter. Thus, as long as the planet's angular diameter is smaller than the angular diameter of the first Airy dark ring ( $48 \mathrm{sec}$ of arc for the CSO at $\lambda=1 \mathrm{~mm}$ ), some coherence is maintained out to the edge of the dish. Although the loss of coherence with increasing radius translates into a loss of sensitivity toward the edge of the aperture, this is acceptable for a strong source such as a planet.

\section{References}

1. W. Sweet, "New Radiotelescopes Open Era of Submillimeter Astronomy," Phys. Today 40, 65-67 (1987).

2. J. C. Bennett, A. P. Anderson, and P. A. McInnes, "Microwave Holographic Metrology of Large Reflector Antennas," IEEE Trans. Antennas Propag. AP-24, 295-303 (1976).

3. P. F. Scott and M. Ryle, "A Rapid Method for Measuring the Figure of a Radio Telescope Reflector," Mon. Not. R. Astron. Soc. 178, 539-545 (1976).

4. D. Morris, "Telescope Testing by Radio Holography," in Proceedings, U.R.S.I. International Symposium on Millimeter and Submillimeter Wave Radio Astronomy, Granada, Spain (1984), pp. 29-50.

5. R. N. Bracewell, The Fourier Transform and Its Applications (McGraw-Hill, New York, 1986).

6. M. P. Godwin, E. P. Schoessow, and B. H. Grahl, "Improvement of the Effelsberg 100 Meter Telescope Based on Holographic Reflector Surface Measurement," Astron. Astrophys. 167, 390 394 (1986).

7. C. E. Mayer, J. H. Davis, W. L. Peters, and W. J. Vogel, "A Holographic Surface Measurement of the Texas 4.9-m Antenna at $86 \mathrm{GHz}$," IEEE Trans. Antennas Propag. AP-32, 102-109 (1983).

8. D. Morris, J. W. M. Baars, H. Hein, H. Steppe, C. Thum, and R. Wohlleben, "Radio-Holographic Reflector Measurement of the 30-m Millimeter Radio Telescope at $22 \mathrm{GHz}$ with a Cosmic Signal Source," Astron. Astrophys. 203, 399-406 (1988).

9. R. Hills and A. Lasenby, "Millimetre-Wave Metrology of the James Clerk Maxwell Telescope," in Proceedings, Eleventh Estec Workshop on Antenna Measurements, Gothenburg, Sweden (1988).

10. D. Morris, "Phase Retrieval in the Radio Holography of Reflector Antennas and Radio Telescopes," IEEE Trans. Antennas Propag. AP-33, 749-755 (1985).

11. P. F. Goldsmith, "Quasi-Optical Techniques at Millimeter and Submillimeter Wavelengths," Infrared Millimeter Waves 6, 277-344 (1982).

12. M. Born and E. Wolf, Principles of Optics (Pergamon, New York, 1980).

13. J. D. Kraus, Radio Astronomy (Cygnus-Quasar, Powell, OH, 1986).

14. J. W. Goodman, Introduction to Fourier Optics (McGraw-Hill, New York, 1968).

15. P. W. Hannan, "Microwave Antennas Derived from the Cassegrain Telescope," IRE Trans. Antennas Propag. 9, 140-153 (1961).

16. J. F. James and R. S. Sternberg, Design of Optical Spectrometers (Chapman \& Hall, London, 1969).

17. T. G. Phillips, "Techniques of Submillimeter Astronomy," in Millimetre and Submillimetre Astronomy, R. D. Wolstencroft and W. B. Burton, Eds. (Kluwer, Dordrecht, The Netherlands, 1988), pp. 1-25.

18. W. L. Wolfe and G. J. Zissis, Eds., The Infrared Handbook (Office of Naval Research, Washington, DC, 1978). 
19. H.W.Schnopper and R. I. Thompson, "Fourier Spectrometers," Methods Exp. Phys. 12A, 491-529 (1974).

20. R. R. Treffers, "Signal-to-Noise Ratio in Fourier Spectroscopy," Appl. Opt. 16, 3103-3106 (1977).

21. D. Malacara, Optical Shop Testing (Wiley, New York, 1978).

22. D. K. Lambert and P. L. Richards, "New Results in the Theory of a Plane Mirror Interferometer," J. Opt. Soc. Am. 68, 11241130 (1978).

23. S. von Hoerner, "Telescope Surface Measurement with Two Feeds," IEEE Trans. Antennas Propag. AP-26, 857-860 (1978).

24. C. E. Mayer, "Microwave Antenna Metrology by Holographic Means," Ph.D. thesis, U. Texas (1983).

25. M. S. Zarghamee and J. Antebi, "Surface Accuracy of Cassegrain Antennas," IEEE Trans. Antennas Propag. AP-33, 828-837 (1985).

26. D. H. Martin, "Polarizing (Martin-Pupplett) Interferometric Spectrometers for the Near- and Submillimeter Spectra," Infrared Millimeter Waves 6, 66-149 (1982).

27. Infrared Laboratories, Inc., Tucson, AZ.
28. D. A. Harper, R. H. Hildebrand, R. Stiening, and R. Winston, "Heat Trap: An Optimized Far Infrared Field Optics System," Appl. Opt. 15, 53-60 (1976).

29. S. E. Whitcomb and J. Keene, "Low Pass Interference Filters for Submillimeter Astronomy," Appl. Opt. 19, 197-198 (1980).

30. M. Halpern, H. P. Gush, E. Wishnow, and V. De Cosmo, "Far Infrared Transmission of Dielectrics at Cryogenic and Room Temperatures: Glass, Fluorogold, Eccosorb, Stycast, and Various Plastics," Appl. Opt. 25, 565-570 (1986).

31. E. E. Russell and E. E. Bell, "Measurement of the Optical Constants of Crystal Quartz in the Far-Infrared with the Asymmetric Fourier-Transform Method," J. Opt. Soc. Am. 57, 341348 (1967)

32. AT, atmospheric transmission software, Airhead Software, Boulder, CO.

33. D. P. Woody, "Gravitational Deflection of the Leighton Telescopes," in Submillimetre Astronomy, G. D. Watt and A. S. Webster, Eds. (Kluwer, Dordrecht, The Netherlands, 1990), pp. 4344.

34. D.P. Woody, Owens Valley Radio Observatory, Caltech; private communication. 